\title{
Infantile osteoarticular tuberculosis misdiagnosed as Bacillus Calmette-Guerin related osteomyelitis
}

\author{
Noppachart Limpaphayom ${ }^{1, *}$, Phatcharapa Osateerakun ${ }^{2}$, Apiradee Theamboonlers ${ }^{3}$, Sumeth Korkong ${ }^{3}$, \\ and Yong Poovorawan ${ }^{3}$ \\ 1 Department of Orthopaedics, Faculty of Medicine, Chulalongkorn University, 10330 Bangkok, Thailand \\ 2 Department of Orthopaedics, King Chulalongkorn Memorial Hospital, Thai Red Cross Society, 10330 Bangkok, Thailand \\ 3 Center of Excellence in Clinical Virology, Department of Pediatrics, Faculty of Medicine, Chulalongkorn University, \\ 10330 Bangkok, Thailand
}

Received 7 May 2015, Accepted 20 June 2015, Published online 21 July 2015

\begin{abstract}
Tuberculosis, a re-emerging public health problem, is uncommon in infancy. Two healthy completely immunized infants presenting with manifestations compatible with osteoarticular infection required surgical debridement. The cultures of the specimens were positive for M. tuberculosis (MTB) complex comprised multiple subspecies. One case was misdiagnosed as a Bacillus Calmette-Guerin (BCG) related osteomyelitis by a polymerase chain reaction (PCR) based on detection of genes at the region of difference 1. Genome extraction and PCR using the rimM gene and sequences analysis against MTB and BCG control samples confirmed that both specimens were infected by M. tuberculosis. The lesions were successfully healed within one year. Surgical debridement of suspected lesions is warranted in infants as a definitive treatment and to obtain tissues for further evaluation. Microbiological cultures only confirm nonspecific MTB complex infection. PCR kits may yield a false positive result. Identification of the pathogen by DNA extraction and sequence analysis should be recommended.
\end{abstract}

Key words: Infant, Mycobacterium bovis, Mycobacterium tuberculosis, Osteomyelitis, Debridement.

The Mycobacterium tuberculosis (MTB) complex infection, a re-emerging public health problem, is uncommon in infancy [1, 2]. The Bacillus Calmette-Guerin (BCG) vaccine, a live attenuated Mycobacterium bovis, provides significant benefits of disease prevention. However, adverse effects of $\mathrm{BCG}$ vaccination have been reported recently [3, 4]. Clinical manifestations of the MTB complex, e.g. M. tuberculosis, $M$. bovis, and $M$. africanum, osteomyelitis are similar and a variety of laboratory tests have been used to identify the pathogen. The recommended treatments are also variable in this age group $[2,4]$.

We report $M$. tuberculosis osteoarticular infection in infants who were successfully treated by surgical debridement. The specimen in one case was misdiagnosed as M. bovis BCG infection using a commercial polymerase chain reaction (PCR) kit. The organism was later confirmed by PCR for the rimM gene and sequence analysis.

\section{Case report}

Two infants presented with the chief complaint of a painful swollen and diminished range of movement of an extremity with an osteolytic lesion at the metaphysis adjacent to the involved joint. Due to the presentations, open debridement and curettage were performed. Clinical details are shown in Table 1. The radiographic studies and intraoperative findings of patient 1 are shown in Figures $1 \mathrm{~A}-1 \mathrm{~J}$. The radiographic study of patient 2 is shown in Figure 2A. The source cases of $M$. tuberculosis infection in the respective family could not be identified. The study was approved by the Institutional Review Board Committee of the Faculty of Medicine, Chulalongkorn University, Thailand (COE 017/2013).

Microbiologic profiles are presented in Table 2. The specimens from both infants had a positive culture for MTB complex. The PCR for BCG using Seeplex ${ }^{\circledR}$ MTB/BCG kit (Seegene, Seoul, Korea) to detect the genes in the region of difference 1 (RD1) was done in patient 2 after a subsequent case of suspected MTB complex infection was presented in an infant who was healthy and showed a positive result for a BCG. M. tuberculosis infection was subsequently confirmed

*Corresponding author: Noppachart.L@chula.ac.th 
Table 1. Demographic and laboratory data of both patients.

\begin{tabular}{|c|c|c|c|}
\hline Patient data & Patient 1 & Patient 2 & Reference range \\
\hline Gender/age (months) & Female/6 & Female/12 & \\
\hline Location/duration & Right talus and ankle joint/ 1 week & Right proximal femur and hip joint/2 weeks & \\
\hline \multicolumn{4}{|l|}{ Physical examination } \\
\hline Body temperature $\left({ }^{\circ} \mathrm{C}\right)$ & 37 & 37 & \\
\hline BCG scar & Left shoulder & Left gluteal & \\
\hline Body weight (kg) & 6.6 & 9.3 & \\
\hline Height $(\mathrm{cm})$ & 68 & 73 & \\
\hline PPD skin test (mm) & 18 & 15 & \\
\hline \multicolumn{4}{|l|}{ Laboratory findings } \\
\hline \multicolumn{4}{|l|}{$\mathrm{CBC}$} \\
\hline $\mathrm{Hb}(\mathrm{g} / \mathrm{L})$ & 110.0 & 127.0 & $120.0-150.0$ \\
\hline Hct (proportion of 1.0 ) & 0.33 & 0.39 & $0.36-0.45$ \\
\hline WBC $\left(\times 10^{9} / \mathrm{L}\right)$ & 9.83 & 17.48 & $4.5-11.0$ \\
\hline Neutrophils (proportion of 1.0 ) & 0.40 & 0.24 & $0.40-0.71$ \\
\hline Lymphocytes (proportion of 1.0 ) & 0.49 & 0.70 & $0.22-0.43$ \\
\hline Monocytes (proportion of 1.0 ) & 0.09 & 0.04 & $0.0-0.07$ \\
\hline Eosinophils (proportion of 1.0 ) & 0.03 & 0.01 & $0.0-0.04$ \\
\hline Basophils (proportion of 1.0 ) & 0.004 & 0.002 & $0.0-0.02$ \\
\hline ESR $(\mathrm{mm} / \mathrm{h})$ & 54 & 9 & $0-28$ \\
\hline C-Reactive protein $(\mathrm{mmol} / \mathrm{L})$ & 331.4 & 55.24 & $<47.62$ \\
\hline Chest X-ray & Normal & Normal & \\
\hline
\end{tabular}

BCG, Bacillus Calmette-Guerin; PPD, purified protein derivative; CBC, complete blood count; Hb, hemoglobin; Hct, hematocrit; WBC, white blood cell; ESR, erythrocyte sedimentation rate.

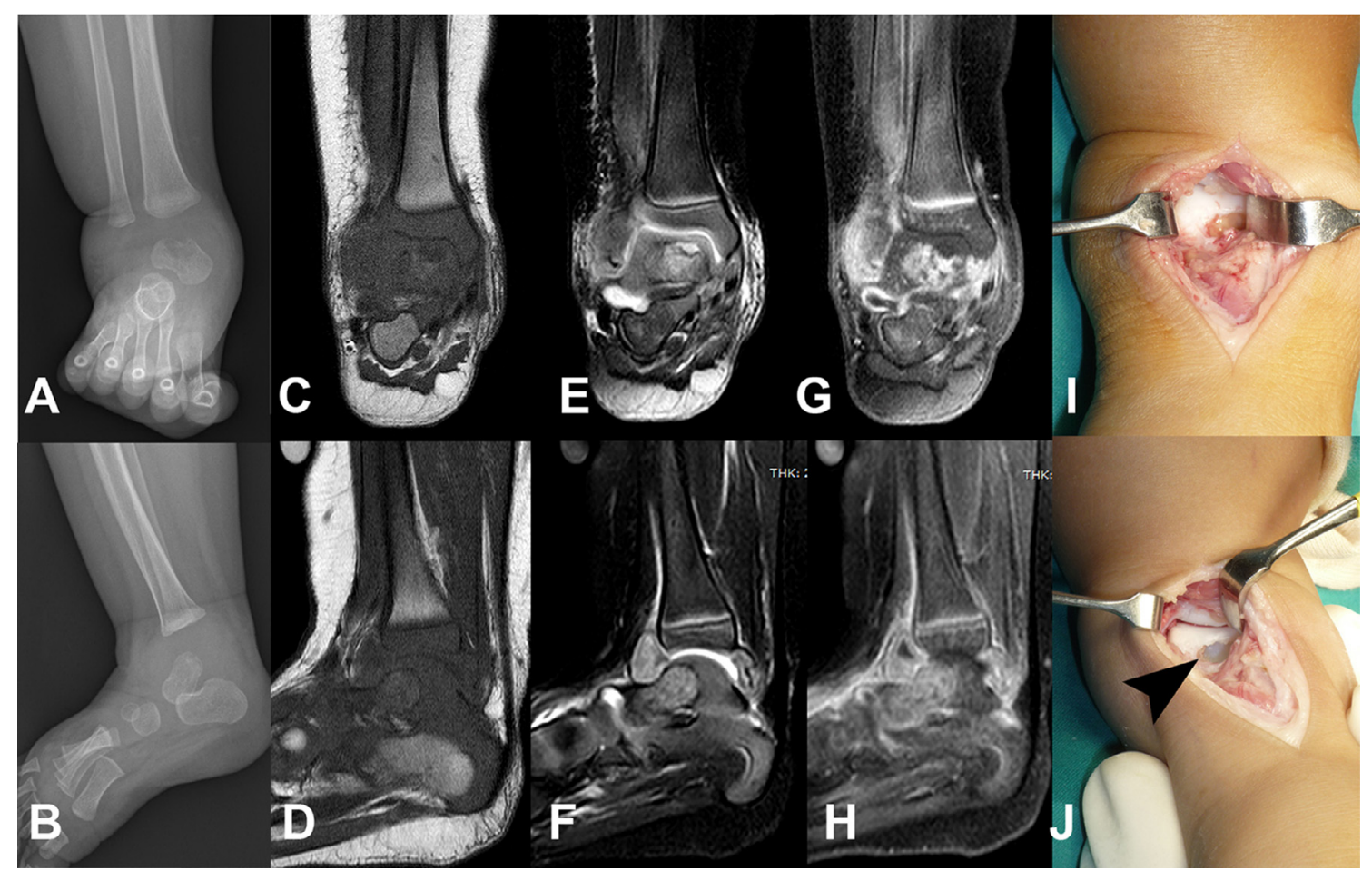

Figure 1. Anteroposterior (A) and lateral (B) radiographs of the right ankle of patient 1 are shown along with magnetic resonance imaging images in $\mathrm{T} 1$ weighted $(\mathrm{C}, \mathrm{D}), \mathrm{T} 2$ weighted $(\mathrm{E}, \mathrm{F})$, and gadolinium-enhanced $(\mathrm{G}, \mathrm{H})$. Intraoperative finding before $(\mathrm{I})$ and after $(\mathrm{J})$ debridement is demonstrated. The arrowhead represents an osteolytic lesion at the talus. 


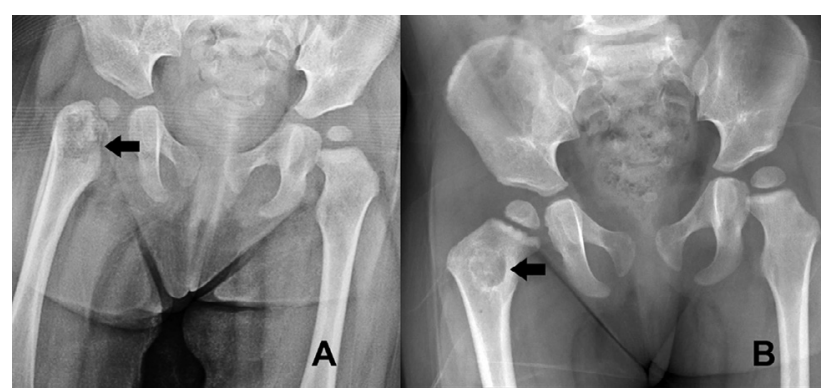

Figure 2. Radiographs of the hips of patient 2 are shown before surgery (A) and at 12-month follow-up visit (B). The arrows represent an osteolytic lesion at the right proximal femur.

by DNA extraction and sequence analysis. A standard 12-month course of anti-tuberculosis regimen including isoniazid, rifampicin, ethambutol, and pyrazinamide was prescribed. Both patients resumed full-weight bearing ability after 3 months. At 12-month follow-up, both infants demonstrate a satisfactory healing radiographically and the radiographs are shown in Figure 2B for patient 1 and Figure 3 for patient 2, respectively.

\section{Detection of MTB and BCG}

The PCR was performed using F3 $5^{\prime}$ CTAAGGG GCCTTTTGACGG $3^{\prime}$ as a sense primer and B3 $5^{\prime}$ CACACTTCGGTGACGACAC $3^{\prime}$ as an antisense primer and was subjected to gel electrophoresis (Figure 4). The amplified product (274 bp) was excised. The nucleotide sequences were analyzed using the BCG vaccine (Queen Saovabha Memorial Institute, Thailand) and the M. tuberculosis culture was used as a positive control.

Nucleotide sequences were compared with the rim $M$ gene of the reference strain of M. Tuberculosis H37Rv (Gene ID: 887188). The alignment between the studied samples and the strain of M. Tuberculosis H37Rv in partial rimM gene showed $100 \%$ identity. The sequences were subjected to BLAST analysis and displayed $99 \%$ sequences homology with the strain obtained from M. tuberculosis EAI5 isolated in Mumbai, India. Chromatograms of nucleotide sequences of the two samples compared with those of $M$. tuberculosis and $M$. bovis BCG control sequences showed the identical sequences with M. tuberculosis (Figure 5).

\section{Discussion}

M. tuberculosis osteoarticular infection in children aged less than 12 months is rare $[1,2]$. Due to the high prevalence of $M$. tuberculosis in the region, the diagnosis is high on the list when evaluating a child with bone and joint pain $[1,4]$. The clinicians require a high index of suspicion, should be familiar with the clinical presentations and aware of other possible causative organisms [4]. The BCG vaccine consisted of at least six BCG substrains and it is variable among countries [5]. The BCG Tokyo-172 strain was utilized in several Asian countries including South Korea, Taiwan, and Thailand, while others, e.g. China and Hong Kong, utilized the Danish strain [1, 3-5]. Osteoarticular infection caused by the BCG vaccine may occur [3, 4].

Clinically, M. tuberculosis and $M$. bovis BCG-related osteomyelitis is indistinguishable [2,3]. Painful swelling of the extremity, decreasing range of motion of an affected joint in the early stage, and no or low grade fever raises a possibility of osteoarticular infection. There has been an increasing awareness of BCG Tokyo-172 strain associated complications which raised concerns regarding its safety and the vaccine is under a surveillance program in some countries [3]. Hematogenous spread would be the most likely explanation since the affected extremity was not limited to the site of the BCG administration [4]. This could be a serious threat to the reputation of the national vaccination program.

The radiographic appearances are usually nonspecific for both organisms including poorly demarcated cystic lesions and minimal sclerotic reactions at the metaphysis or the epiphysis [4]. The joint space is preserved in the early stage and gradually damaged by indirect subchondral erosion and pannus formation [2]. Synovitis, joint effusion, and epiphyseal and metaphyseal involvement are common findings shown on MRI images. Additionally, an MRI is a useful investigation for a preoperative planning [4]. Pathological examination usually shows nonspecific granulomatous osteomyelitis. Moreover, an MTB complex culture comprises multiple organisms. Other pathogens, e.g. M. bovis $\mathrm{BCG}$, should be tested for when an apparent MTB complex infection occurs in an infant with a successful immunization confirmed by a visible BCG scar and a positive tuberculin skin test but unidentifiable $M$. tuberculosis source cases [4].

Although anti-tuberculosis chemotherapies remain a principal treatment, pyrazinamide, a recommended first line treatment of $M$. tuberculosis infection, is not effective against an $M$. bovis, thus early differentiation between the two pathogens by a reliable test is crucial. While chemotherapeutic treatment without surgical debridement of the lesion was suggested, this could be challenging in a certain age group. The fine needle aspiration was found to yield a positive result in $1 / 3$ to $2 / 3$ of cases [4]. By contrast, our treatment is justified by presentations of infants, an age group where verbal communication is inadequate, and the high prevalence of M. tuberculosis $[1,2,4]$. Surgical debridement was recommended to improve the general condition of the infant and remove necrotic tissue. This allows chemotherapeutic agents to eradicate the MTB sequestrated in the osteolytic lesion and a specimen is obtained to ascertain a diagnosis in a patient from an area endemic for bacterial and fungal infections [2, 4]. Our experience showed that the osteolytic lesion healed satisfactorily at one year as shown on the radiograph.

The usefulness of the PCR method to differentiate between M. tuberculosis and BCG has been reported [3, 4]. Genes in the $\mathrm{RD} 1$ region produce proteins responsible for the virulence of M. tuberculosis and they are present in every strain of M. tuberculosis but by contrast, are absent in the BCG [5]. Our result demonstrates that a commercial PCR kit can potentially produce a false positive result. The $\operatorname{rim} M$ gene is the $16 \mathrm{~S}$ rRNA-processing protein gene and the sequence is specific for M. tuberculosis and M. bovis. We choose to study genes in difference genomic region to avoid a false positive result 
Table 2. Results of bacteriological investigations of both patients.

\begin{tabular}{lcc}
\hline & Patient 1 & Patient 2 \\
\hline $\begin{array}{l}\text { Surgical specimen } \\
\text { Aerobic culture }\end{array}$ & No growth & No growth \\
Mycobacterium culture & Positive for Mycobacterium & tuberculosis complex \\
Gram stain & No organism & $\begin{array}{c}\text { Positive for Mycobacterium } \\
\text { tuberculosis complex } \\
\text { AFB stain }\end{array}$ \\
Positive for AFB stain & No organism \\
Gastric lavage culture & No growth & Negative for AFB stain \\
Hemoculture & No growth & No growth \\
Pathological examination & N/A & No growth \\
PCR for BCG by commercial kit & Granulomatous osteomyelitis & Granulomatous osteomyelitis \\
\hline
\end{tabular}

AFB, acid-fast bacilli; PCR, polymerase chain reaction; BCG, Bacillus Calmette-Guerin; N/A, not available.

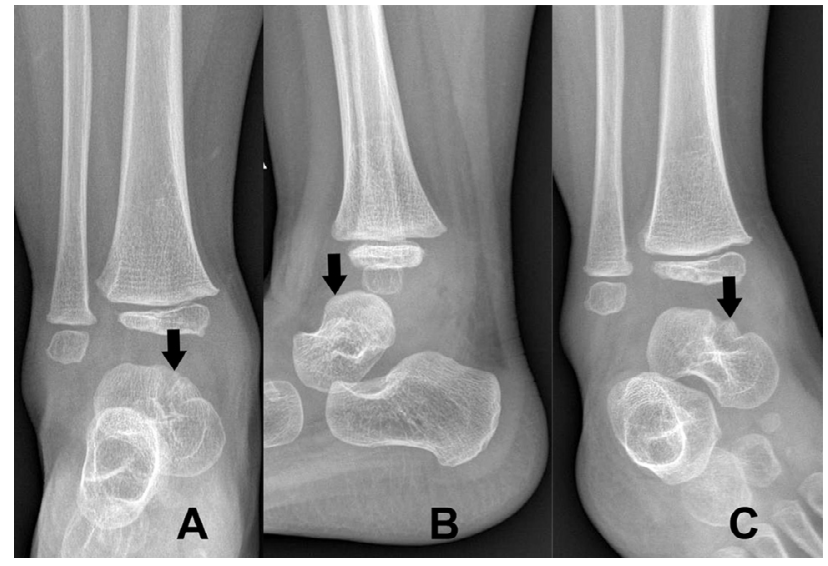

Figure 3. Radiographs of patient 1 at 12 -month follow-up are demonstrated. The arrows represent an osteolytic lesion at the talus.

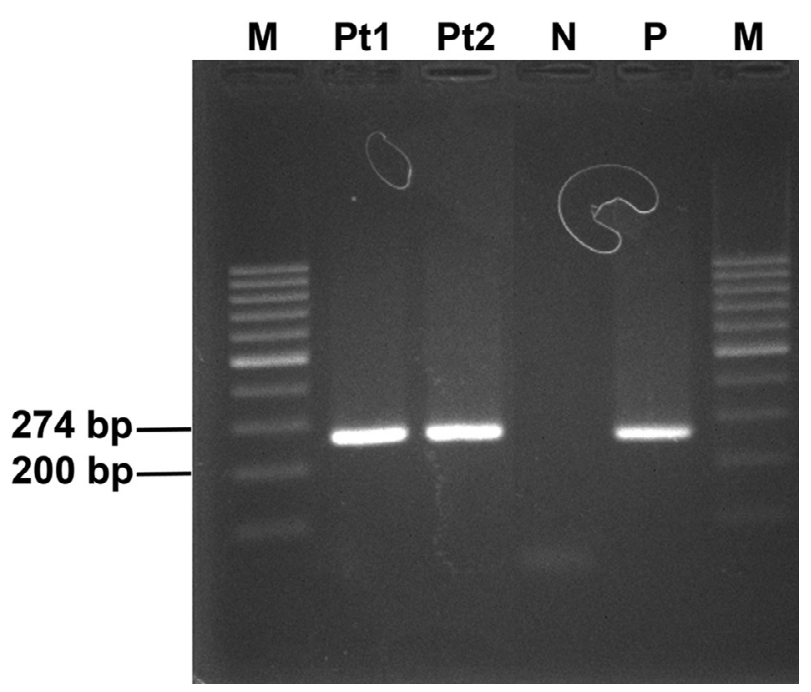

Figure 4. Electrophoresis of the polymerase chain reaction products generated a band of $274 \mathrm{bp}$ in good agreement with the expected size. M,100 bp marker; Pt1, sample from patient 1; Pt2, sample from patient $2 ; \mathrm{N}$, negative control; and $\mathrm{P}$, positive control.

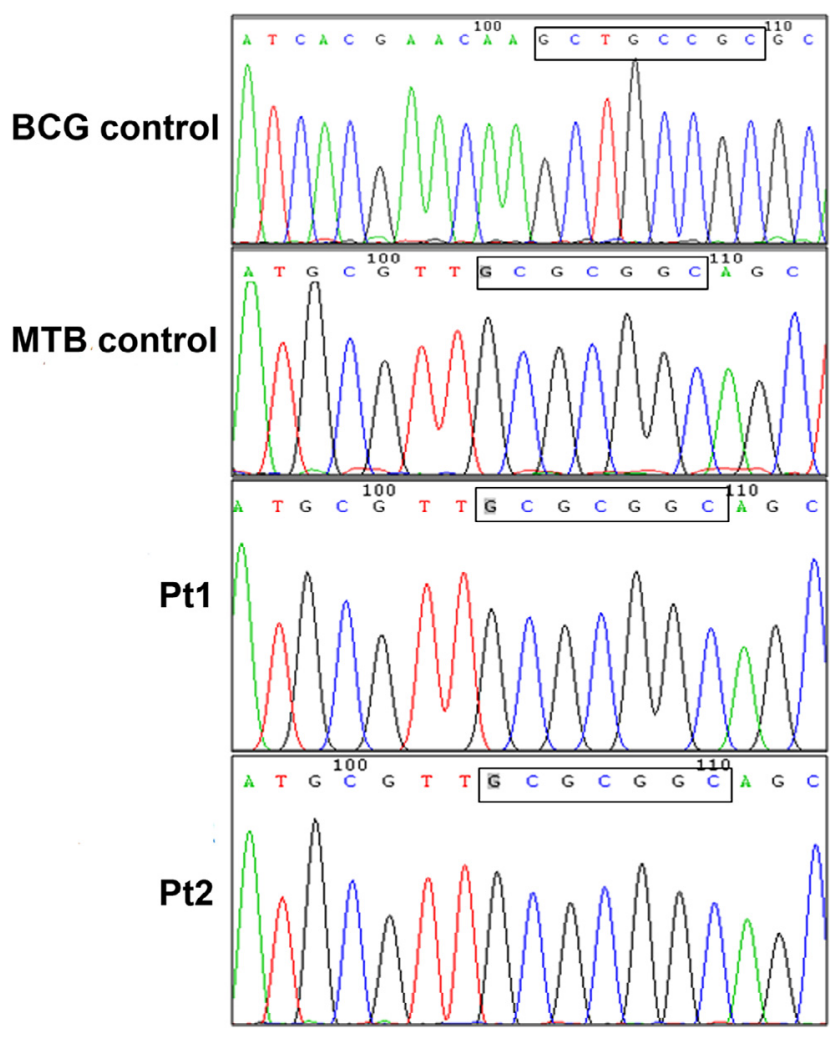

Figure 5. Chromatograms of nucleotide sequences of the two positive samples compared with Mycobacterium tuberculosis and Bacillus Calmette-Guerin control samples showing sequences identical with Mycobacterium tuberculosis. MTB, Mycobacterium tuberculosis; BCG, Bacillus Calmette-Guerin; Pt1, sample from patient 1 ; Pt2, sample from patient 2.

and found that our samples contained either mycobacterium species which were later confirmed to be M. tuberculosis by sequence analysis. The most frequent explanation for false positive PCR results is sample contaminations; but this is very unlikely since the BCG is not a routine organism being tested. We recommend that the investigations should include a 
complete set of investigation for all possible organisms based on the incidence and geographical zone [2,4].

In conclusion, prompt surgical debridement of an osteolytic lesion suspected of being an infection is warranted in an infant. Differentiation between $M$. tuberculosis and M. bovis BCG-related infection in those who have received BCG vaccination should be performed when an M. tuberculosis source is unidentifiable. A specimen culture is the gold standard for mycobacterial infection but provides only nonspecific result. The PCR kit could be used as a screening test for $M$. bovis BCG infection. DNA extraction and sequence analysis is more time-consuming but could be recommended as a confirmation method.

\section{Conflict of interest}

The authors, NL, PO, AT, SK, and YP, certify that they have no financial conflict of interest in connection with this article. No benefits in any form have been received or will be received from a commercial party related directly or indirectly to the subject of this article.

Acknowledgements. This work is supported by the Higher Education Research Promotion and National Research University Project of Thailand, Office of the Higher Education Commission (HR 1155A-55), Thailand Research Fund (DPG5480002), the Center of Excellence in Clinical Virology, Chulalongkorn University,
Integrated Innovation Academic Center IIAC Chulalongkorn University Centenary Academic Development Project (CU56HR01), and the Ratchadaphiseksomphot Endowment Fund of Chulalongkorn University (RES560530093).

The authors would like to thank the staff of the Department of Microbiology, Faculty of Medicine, Chulalongkorn University and King Chulalongkorn Memorial Hospital for their generous support on culture of the bacteria.

\section{References}

1. World Health Organization (2012) Tuberculosis Control in the South-East Asia 2012. http://whothailand.healthrepository. org/bitstream/123456789/1430/1/2012\%20Report-Tuberculosis\% 20Control\%20in\%20SE\%20Asia\%20Region.pdf. Accessed June 7, 2013.

2. Rasool MN (2001) Osseous manifestations of tuberculosis in children. J Pediatr Orthop 21(6), 749-755.

3. Jou R, Huang WL, Su WJ (2009) Tokyo-172 BCG vaccination complications, Taiwan. Emer Infect Dis 15(9), 1525-1526.

4. Yoo WJ, Choi IH, Yun YH, et al. (2014) Primary epiphyseal osteomyelitis caused by mycobacterium species in otherwise healthy toddlers. J Bone Joint Surg Am 96(17), e145.

5. Lewis KN, Liao R, Guinn KM, et al. (2003) Deletion of RD1 from Mycobacterium tuberculosis mimics bacille CalmetteGuérin attenuation. J Infect Dis 187(1), 117-123.

Cite this article as: Limpaphayom N, Osateerakun P, Theamboonlers A, Korkong S \& Poovorawan Y (2015) Infantile osteoarticular tuberculosis misdiagnosed as Bacillus Calmette-Guerin related osteomyelitis. SICOT J, 1, 20 